\title{
EDITORIAL
}

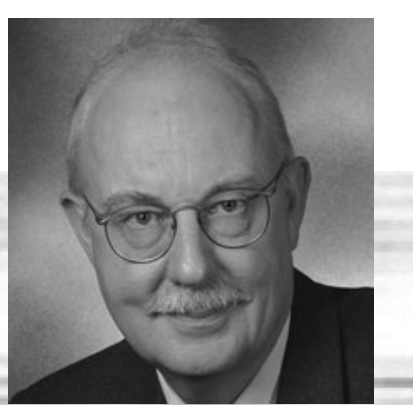

C. Müller-Schloer

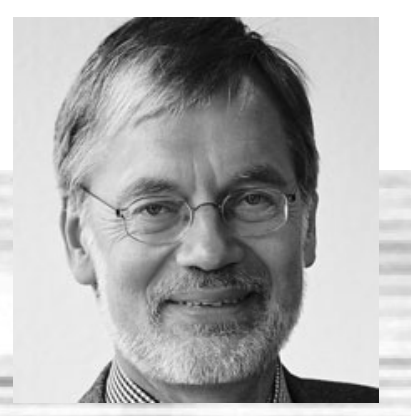

H. Schmeck

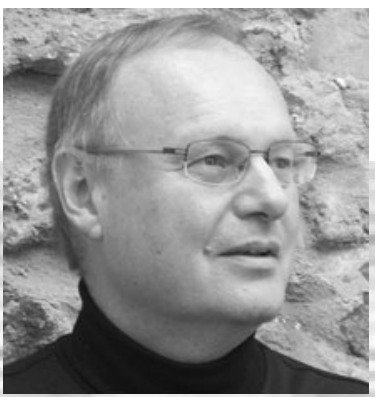

T. Ungerer

\section{Organic Computing}

Organic Computing (OC) hat sich das Ziel gesetzt, Lösungen für zukünftige hochkomplexe IT-Systeme und deren Beherrschung zu entwickeln. Komplexität entsteht durch die sehr große Anzahl von Komponenten und Subsystemen solcher Systeme, durch deren zunehmende Vernetzung und damit wechselseitige Abhängigkeit, durch die Offenheit künftiger Systeme sowie durch die prinzipielle Unmöglichkeit einer exakten Vorhersage aller künftigen Ziele und Störeinflüsse. Veränderungen sind nicht die Ausnahme, sie sind die Regel. Und diese permanente Veränderung ist keine Eigenschaft, welche wir diesen Systemen ohne Not verleihen. Veränderung ist unvermeidbar. Als Kernthese des OC wurde daher formuliert:

Es ist nicht die Frage, ob adaptive und selbstorganisierende Systeme entstehen, sondern wie wir sie beherrschen.

Auch wenn Organic Computing noch eine junge Forschungsdisziplin ist, so kann es doch bereits auf eine ansehnliche Geschichte zurückblicken. Nach dem in mehreren Workshops in den Jahren 2002 und 2003 erarbeiten Positionspapier der GI und ITG [1] hat die OC-Gemeinschaft vielfältige Aktivitäten entwickelt. Das ursprünglich noch sehr visionäre Konzept, welches als "Komplexitätsbeherrschung durch Selbstorganisation" umschrieben werden kann, wurde in einer Vielzahl von Forschungsprojekten konkretisiert und mit Leben gefüllt. Die zentrale Rolle spielte dabei das DFGSchwerpunktprogramm 1183 Organic Computing, in dem von 2005 bis 2011 in drei Zweijahresphasen jeweils etwa 20 Projekte gefördert wurden. Das Schwerpunktprogramm wurde im September 2011 abgeschlossen. Die Ergebnisse sind im „OC Compendium" auf 627 Seiten dokumentiert [2].

Das Schwerpunktprogramm Organic Computing verfolgte das Ziel, Konzepte zur Beherrschung zukünftiger hochkomplexer Informations- und Kommunikationssysteme zu entwickeln. Hierzu wurden zentrale Begriffe wie Adaptivität, Autonomie, Robustheit, Flexibilität, Selbstorganisation und Emergenz formal untersucht, um das theoretische Problemverständnis zu vertiefen. Zudem lag ein besonderer Fokus auf Architekturen und Methoden für den Systementwurf, sowie auf deren Umsetzung in konkreten Anwendungsszenarien. Unter Architekturgesichtspunkten spielt die im Schwerpunktprogramm entwickelte generische Observer/Controller-Architektur eine zentrale Rolle. Sie unterstützt die gesteuerte Selbstorganisation technischer Systeme und kommt inner- und außerhalb des Schwerpunkts in verschiedenen Anwendungen erfolgreich zum Einsatz. Weitere im Schwerpunkt entwickelte Architekturansätze umfassen organische Middleware-Systeme, verifizierbare Entwurfsmuster und fehlertolerante Robotikarchitekturen. Für viele der entwickelten Architekturen sind maschinelle Lernverfahren bedeutsam, da sie eine autonome Anpassung der organischen Systeme an sich dynamisch verändernde Anforderungen der Einsatzumgebung unterstützen. Arbeiten in diesem Bereich behandeln den Wissenstransfer zwischen Agenten ebenso wie Konzepte zum Lernen in performanz- und sicherheitskritischen Umgebungen. Darüber hinaus wurden etablierte Lernverfahren wie Learning Classifier Systeme erfolgreich erweitert und verbessert. Die entwickelten Methodiken wurden u. a. in dynamisch rekonfigurierbaren, fehlertoleranten Hardware-Plattformen, in adaptiven Verkehrssystemen und in Produktionsszenarien eingesetzt. 


\section{$\{$ EDITORIAL}

Eine Vielzahl von OC-Aktivitäten auch außerhalb des Schwerpunktprogramms dokumentiert die Lebendigkeit des Arbeitsgebiets. Es existieren mehrere Nachfolgeprojekte mit DFG- und BMBF-Förderung, in denen OC-Methoden eingesetzt und weiterentwickelt werden. OC konnte in den sechs Jahren des Schwerpunktprogramms auch im internationalen und im nicht-universitären Bereich etabliert werden. So wurde das Themenfeld Organic Computing fester Bestandteil wichtiger internationaler Konferenzen wie der International Conference on Architecture of Computing Systems (ARCS), der International Conference on Autonomic and Trusted Computing (ATC), der International Conference on Biologically Inspired Cooperative Computing (BICC) und der International Conference on Autonomic Computing (ICAC). Darüber hinaus wurde das Forschungsfeld durch eine Vielzahl von Workshops, Special Sessions und Tutorials im Rahmen weiterer Tagungen repräsentiert. Die internationale Vernetzung führender Wissenschaftler wurde durch die Organisation von Dagstuhl-Seminaren in den Jahren 2006 (Organic Computing - Controlled Emergence), 2008 (Organic Computing - Controlled Self-organization) und 2011 (Organic Computing - Design of Self-Organizing Systems) kontinuierlich gefördert. Zudem wurde eine IEEE Computational Intelligence Society (IEEE CIS) Task Force ins Leben gerufen, die auf eine verbesserte Sichtbarkeit des Themenfelds Organic Computing hinarbeitet. Weiterhin befindet sich in den Fachbereichen Technische Informatik der GI und der ITG die Gründung einer Fachgruppe Organic Computing in Vorbereitung.

Besonders erfreulich ist, dass mittlerweile bereits drei Organic-Computing-Professuren in Deutschland existieren.

OC befindet sich nach Abschluss des Schwerpunktprogramms in einer entscheidenden Phase. Wir sehen zwei wesentliche einander ergänzende Ausrichtungen der künftigen Arbeit:

1. OC soll verstärkt in konkreten Anwendungen zum Einsatz kommen, und

2. es besteht auf mehreren Spezialgebieten des OC noch großer Forschungsbedarf.

Besonders interessante Anwendungsgebiete, für die z. T. auch schon prototypische Lösungen existieren, sind Verkehrstechnik, Kommunikationsnetze, Smart-Grid-Techniken, Fabrikautomatisierung sowie Robotik. Forschungsschwerpunkte des OC werden gesehen im Bereich der Selbst-Optimierung (zur Laufzeit), der kooperativen und institutionellen Strukturen (Social OC) und der kognitiven Architekturen. Weiterhin fehlen noch weitgehend systematische Ansätze zum Entwurfsprozess von OC-Systemen, welcher sich grundlegend von dem herkömmlicher Systeme unterscheidet, sowie zur Einbeziehung des menschlichen Nutzers in das OC-System.

Wir haben uns für den Themenschwerpunkt in diesem Heft des Informatik-Spektrums auf Arbeiten konzentriert, welche außerhalb des Schwerpunktprogramms laufen und somit wegweisend für die weitere Entwicklung des Organic Computing sind. Dabei kann selbstverständlich keine vollständige Abdeckung aller interessanten aktuellen OC-Projekte erreicht werden. Die Artikel haben alle einen eindeutigen Anwendungsbezug. Sie zeigen, dass OC-Techniken praktisch einsetzbar sind. Die Anwendungen liegen in den Bereichen Networks, Robotik, Sicherheit, Smart Grid, Desktop Grid Computing und Gesichtserkennung. Darüber hinaus werden auch grundsätzliche Fragen des OC angesprochen, welche für die weitere Forschung richtungweisend sein können: Dies sind (1) der Einsatz von Methoden der statistischen Mechanik (I. Scholtes), (2) die Einführung explorativen Verhaltens von OC-Systemen (P. Nelson) und (3) die Nutzung sozialer Prinzipien wir Vertrauen und Reputation für die Selbstorganisation technischer Systeme (J.-P. Steghöfer). - Im Einzelnen:

- Ingo Scholtes et al. von der ETH Zürich schlagen in ihrem Beitrag „Organic Design of Massively Distributed Systems: A Complex Networks Perspective" die Nutzung der Random Graph Theory nicht nur zur Analyse bestehender Netzwerke sondern auch als konstruktive Maßnahme vor, um zur Laufzeit Netzwerkstrukturen (Overlays) mit definierten Eigenschaften wie Konnektivität, Fehlertoleranz, Durchmesser etc. zu generieren.

- Phyllis Nelson et al. von der California State Polytecnic University in Pomona, CA, berichten im Beitrag "Cautionary Tales: Lessons Learned About Unanticipated Behaviors in OC Systems" über unerwartete Effekte bei der Implementierung von OC-Mechanismen in realen Robotik-Anwendungen und schlagen den Einbau eines "opportunistischen Explorationsverhaltens" vor, welches es den Robotern - in Analogie zu Lebewesen - ermöglicht, ihre Welt- und Selbstmodelle aktiv zu verbessern. 
- Jörg Hähner et al. (Leibniz Universität Hannover) zeigen in ihrem Beitrag „Selbstorganisierende Smart-KameraSysteme" auf, wie OC-Techniken genutzt werden können, um ohne eine zentrale Instanz eine fortlaufende Optimierung der räumlichen Partitionierung auch unter Störungen wie dem Ausfall von Kameras zu erreichen.

- Andreas Kamper et al. (Cirquent GmbH bzw. KIT) diskutieren im Beitrag „Adaptives verteiltes Lernen in Bilanzkreisen" die Anwendung mehrstufiger Observer/Controller-Strukturen für den selbstorganisierenden Lastausgleich in Smart-Grid-Systemen und zeigen, dass sich so Erzeuger/Verbraucher-Ungleichgewichte deutlich reduzieren lassen. - Rolf Würtz et al. von der Ruhr Universität Bochum berichten in ihrem Beitrag „Lernen situationsunabhängiger Personenerkennung" über den Einsatz von OC-inspirierten Lerntechniken zur automatischen Gesichtserkennung, deren Erkennungsgüte zum Teil über der menschlicher Versuchspersonen liegt.

- Jan-Philipp Steghöfer et al. von der Universität Augsburg diskutieren im Beitrag „Die Guten, die Bösen und die Vertrauenswürdigen (Vertrauen im Organic Computing)" die Nutzung von Vertrauenskonzepten wie funktionale Korrektheit, Betriebssicherheit, Datensicherheit, Zuverlässigkeit, Glaubwürdigkeit und Gebrauchstauglichkeit für selbstorganisierende Energienetze und offene Desktop Grid Computing Systeme.

Wir möchten den Autoren für die fristgerechte Fertigstellung und dem Review-Team ${ }^{1}$ für die kritische Durchsicht der Beiträge danken.

\section{Müller-Schloer \\ H. Schmeck \\ T. Ungerer}

\section{Literatur}

1. Allrutz R, Cap C, Eilers S, Fey D, Haase H, Hochberger C, Karl W, Kolpatzik B, Krebs J, Langhammer F, Lukowicz P, Maehle E, Maas J, Müller-Schloer C, Riedl R, Schallenberger B, Schanz V, Schmeck H, Schmid D, Schröder-Preikschat W, Ungerer T, Veiser H-0, Wolf L (2003) VDE/ITG/GI - Positionspapier Organic Computing: Computer- und Systemarchitektur. Gesellschaft für Informatik e.V.

2. Müller-Schloer C, Schmeck H, Ungerer T (2011) Organic Computing: A Paradigm Shift for Complex Systems, Birkhäuser Verlag, http://www.springerlink. com/content/978-3-0348-0129-4/contents/

\footnotetext{
${ }^{1}$ Die Durchsicht der Beiträge haben dankenswerterweise die folgenden Kolleginnen und Kollegen übernommen: Prof. Dr. J. Branke,
} Prof. Dr. U. Brinkschulte, Prof. Dr. J. Hähner, Prof. Dr. A. Herkersdorf, Prof. Dr. W. Karl, Dr. S. Mostaghim, Prof. Dr. G. Mühl, Prof. Dr. B. Sick, Prof. Dr. J. Teich, Dr. R. Würtz. 